\title{
Cryogenic Thermal Distortion Performance Characterization for the JWST ISIM Structure
}

\author{
John Johnston* ${ }^{\mathrm{a}}$, Emmanuel Cofie ${ }^{\mathrm{b}}$, Jason Hylan ${ }^{\mathrm{a}}$, Raymond Ohl ${ }^{\mathrm{a}}$, Maria Nowak ${ }^{\mathrm{a}}$, Douglas \\ McGuffey ${ }^{a}$, James Pontius ${ }^{a}$, Eric Johnson ${ }^{a}$ \\ ${ }^{a}$ NASA Goddard Space Flight Center, Greenbelt, MD, USA 20711 \\ ${ }^{b}$ SGT, Inc., Seabrook, MD, 20706
}

\begin{abstract}
The James Webb Space Telescope (JWST) Integrated Science Instrument Module (ISIM) Structure is a precision optical metering structure for the JWST science instruments. Optomechanical performance requirements place stringent limits on the allowable thermal distortion of the metering structure between ambient and cryogenic operating temperature ( 35 $\mathrm{K})$. This paper focuses on thermal distortion testing and successful verification of performance requirements for the flight ISIM Structure. The ISIM Structure Cryoset Test was completed in Spring 2010 at NASA Goddard Space Flight Center in the Space Environment Simulator Chamber. During the test, the ISIM Structure was thermal cycled twice between ambient and cryogenic ( $\sim 35 \mathrm{~K})$ temperatures. Photogrammetry was used to measure the Structure in the ambient and cryogenic states for each cycle to assess both cooldown thermal distortion and repeatability. This paper will provide details on the post-processing of the metrology datasets completed to compare measurements with performance requirements.
\end{abstract}

Keywords: JWST, ISIM Structure, Cryogenic Performance Test, Thermal Distortion, Photogrammetry

\section{INTRODUCTION}

\subsection{Overview of JWST, ISIM, and the ISIM Structure}

The James Webb Space Telescope (JWST) is a large, infrared space telescope consisting of an optical telescope element (OTE), an integrated science instrument module (ISIM), a spacecraft, and a sunshield (Figure 1). ${ }^{1}$ ISIM consists of the JWST science instruments (NIRCam, MIRI, NIRSpec), a fine guidance sensor (FGS), the ISIM Structure, and thermal and electrical subsystems (Figure 1). ${ }^{2}$ The ISIM Structure is an approximately $2 \mathrm{~m} \mathrm{x} 2 \mathrm{~m} \mathrm{x} 1.5 \mathrm{~m}$ bonded composite frame that acts as a metering structure between the instruments/guider and the telescope. ${ }^{3,4}$ The ISIM Structure interfaces to the OTE via a kinematic mount (KM) system consisting of two bipods and two monopods. The ISIM Structure and the supported instruments are designed to operate at cryogenic temperatures $(\sim 35 \mathrm{~K})$, and thermal distortion performance is critical to maintaining the alignment of the instruments to the ISIM Structure. Significant effort has been expended on the development of capabilities to predict and measure cryogenic thermal distortion in order to ensure that the associated performance requirements are met.

\subsection{ISIM Structure Cryoset Test}

ISIM Structure development and verification followed a building blocks approach starting with the development and testing of material samples, followed by bonded joint and subassembly level activities, and culminating with the structural verification program for the flight hardware. Two major cryogenic environmental tests were completed as part of ISIM Structure verification. The Cryoset Test was completed in May 2010 for thermal distortion performance characterization, and the Cryoproof Test was completed in October 2010 for demonstration of cryogenic strength. During both these tests, the ISIM Structure was thermal cycled between ambient and cryogenic temperatures with metrology performed via photogrammetry at the warm and cold states. The test was completed in the NASA Goddard Space Environment Simulator (SES) thermal vacuum chamber, which has been outfitted with a new Helium shroud to achieve the required cryogenic temperature test environment.

*John.D.Johnston@nasa.gov; phone 301-286-2836; fax 301-286-2696; www.nasa.gov, www.jwst.nasa.gov. 
The test configuration for the Cryoset Test, Figure 2, consists of the bare flight ISIM Structure mounted to mechanical ground support equipment (MGSE) which interfaces with the thermal vacuum chamber. The most critical MGSE hardware from a distortion standpoint is the ISIM Test Platform (ITP) which acts as a surrogate for the flight telescope interface during ISIM Element level testing. Metrology targets are attached to key interfaces and tracking locations on the flight hardware and optomechanical MGSE for measurement via the photogrammetry system during testing. Details of the photogrammetry system, its error budgets, and performance will not be discussed in detail here as they have been previously reported $^{5,6}$ This paper summarizes the post-processing of metrology data from the ISIM Structure Cryoset test for cooldown thermal distortion performance verification. Additionally, photogrammetry data was used to validate the thermal distortion model predictions for cooldown performance between room temperature and cryogenic operating temperature. $^{7}$
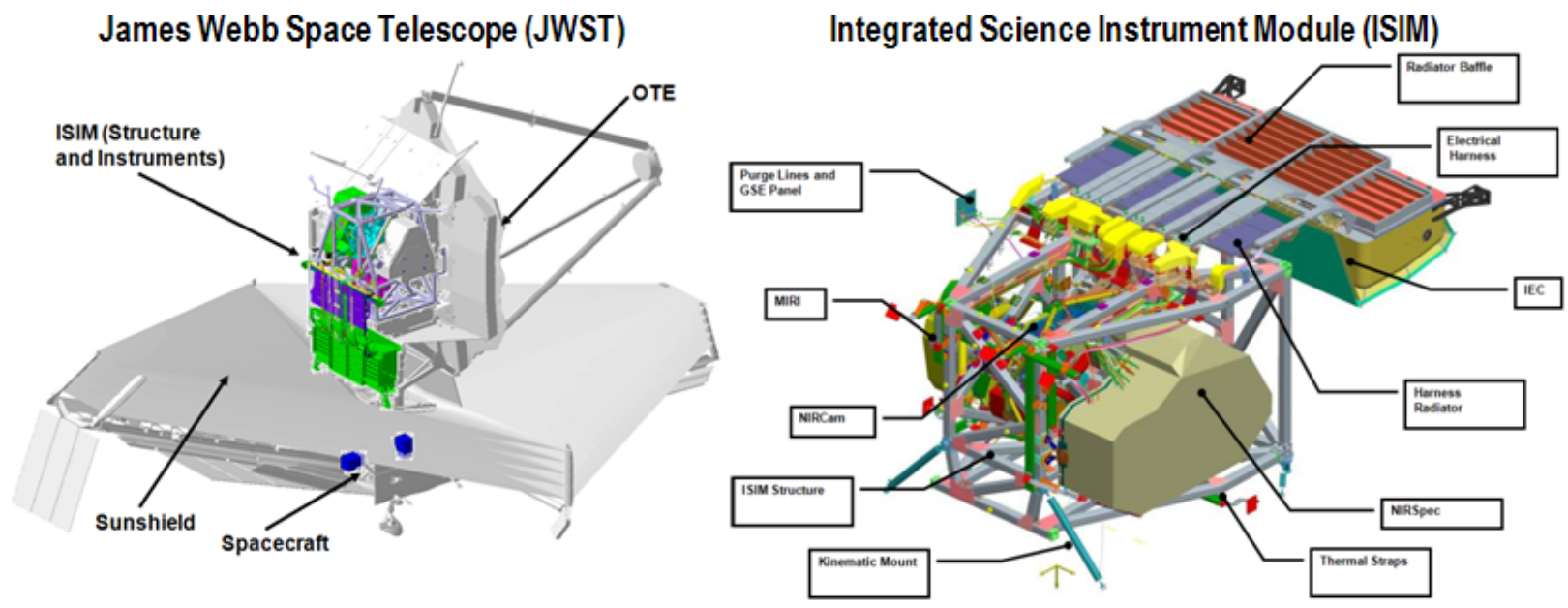

Figure 1. James Webb Space Telescope (JWST) and Integrated Science Instrument Module (ISIM).
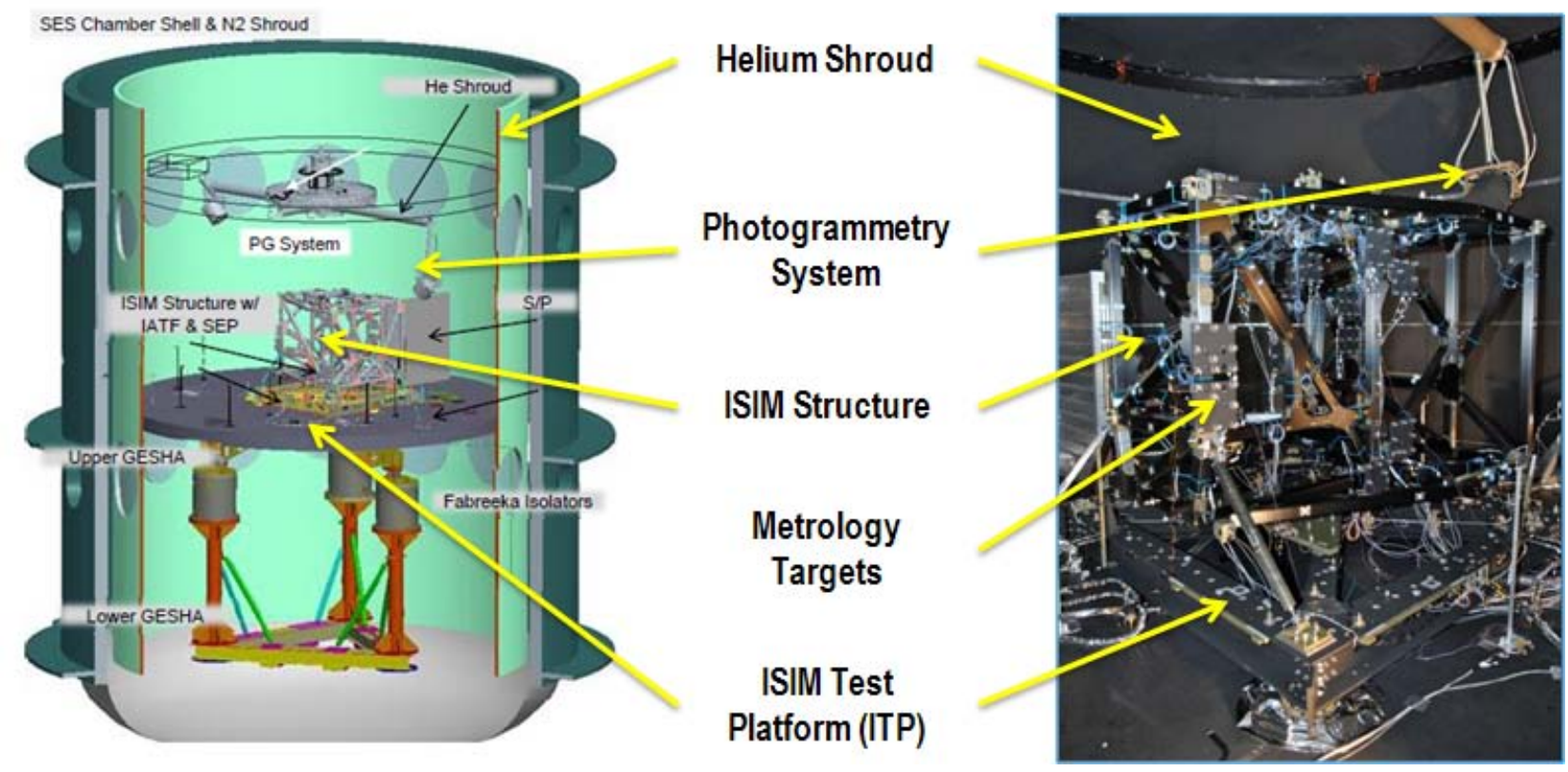

Figure 2. ISIM Structure Cryoset Test Configuration 


\subsection{Overview of paper}

This paper will describe the process by which metrology data from the ISIM Structure Cryoset test was post-processed for use in comparing performance with requirements. First, the ISIM optomechanical coordinate systems and performance requirements are described. Next, the post-processing steps completed are summarized. Finally, postprocessed results are compared to performance requirements demonstrating the ISIM Structure meets cooldown performance requirements.

\section{COORDINATE SYSTEMS}

There are two ISIM-centric optomechanical coordinate systems referenced in the ISIM Structure optomechanical performance requirements. The ISIM coordinate systems are parallel to and offset from the primary coordinate system for the JWST observatory, the "V-coordinate system" (Figure 3). For verification of requirements, metrology based references/coordinate systems were used as described below.

\subsection{Optomechanical Coordinate System A}

Optomechanical coordinate system A (OMCS A) represents the ISIM-to-OTE interface, with Reference plane A at the bottom of the ISIM kinematic mounts. There are 18 "A" targets on the ITP that represent the best measurement of the ISIM-to-OTE interface. Reference ACG motions are calculated based on motions of the 18 A targets on the ITP. Translations are based on the average of the 18 A target translations (thus, the notation "ACG” or "A center of gravity"). Rotations are calculated about ACG as the center for rotations are based on least-squares fit of all 18 A targets. Motions of References A and ACG are defined as zero for comparison of nominal performance with requirements.

\subsection{Optomechanical Coordinate System B}

Optomechanical coordinate system B (OMCS B) represents the ISIM Structure, with Reference plane B at the top of the ISIM kinematic mounts. The 8 "B" targets on the ISIM Structure represent the best measurement of the ISIM Structure's rigid body motion. Reference BCG motions are calculated based on motions of the $8 \mathrm{~B}$ targets on the Structure. Translations are based on the average of the $8 \mathrm{~B}$ target translations. Rotations are calculated about BCG as the center for rotations are based on least-squares fit of all $8 \mathrm{~B}$ targets.
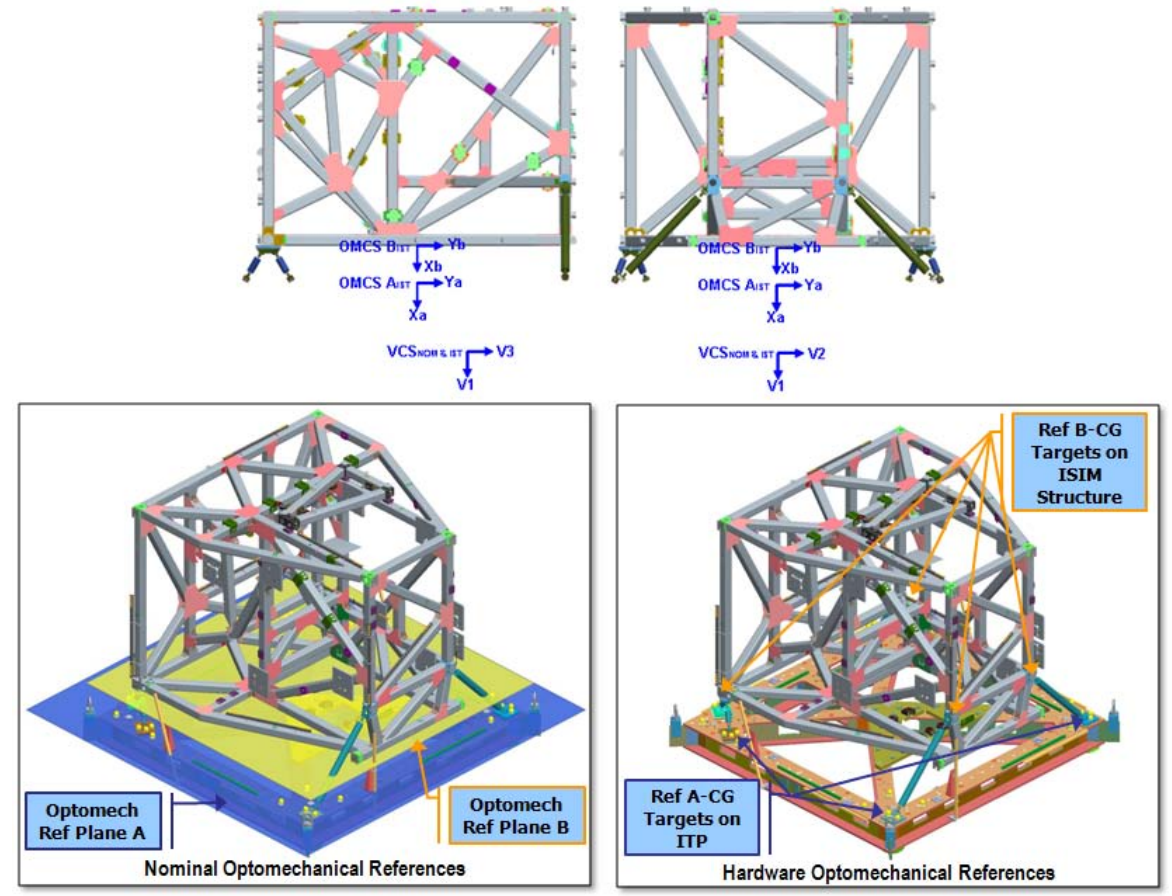

Figure 3. ISIM Optomechanical References. 


\section{OPTOMECHANICAL PERFORMANCE REQUIREMENTS}

ISIM Structure optomechanical requirements for cooldown thermal distortion performance are summarized here. The optomechanical requirements are in terms of: (a) Motion of OMCS B relative to OMCS A which is representative of rigid body motion of ISIM Structure on KMs with respect to the nominal OTE structure and (b) Motion of science instrument interfaces ("SI Pads") relative to OMCS B which is representative of distortion of ISIM Structure.

\subsection{B-to-A Cooldown Performance Requirements}

There are two cooldown performance requirements for B-to-A motions. The first requirement for B-to-A, Table 1, defines the cooldown motion limit for Reference B relative to Reference A (i.e. rigid body motion of ISIM Structure on KMs). Reference A is defined as a perfect (undeformed) OTE/ISIM interface, independent of temperature, for the purposes of optomechanical requirements. This requirement limits the amount of rigid body motion the SIs may move as a group when cooled to the operating temperature with respect to the OTE, due to ISIM-related effects. The effect of deformations at the OTE/ITP interface is primarily to move the ISIM as a rigid body on its kinematic mounts (B-to-A motion), and is covered under OTE alignment budgets. Actual OTE/ISIM interfaces (both OTE and ITP) deform during cooldown, and interface strain contributions to B-to-A motion must be backed-out to compare predictions and measurements with ISIM level requirements. The back-out process for B-to-A motions completed during postprocessing will be described in Section 4.1. The expected contraction of the ITP upon cooling decreases the amount of V1 motion observed in the ITP-ISIM configuration relative to a nominal, unchanging OTE structure. The ITP ground support equipment also impacts other degrees of freedom, such as $\theta 2$ rotation. Note that when comparing measured performance against this requirement, consideration of the measurement uncertainty is required.

Table 1. B-to-A Cooldown Motion Limit Requirement.

\begin{tabular}{|c|c|c|c|c|c|c|}
\hline \multirow{2}{*}{ BCD Motion of BCG wrt ACG } & \multicolumn{3}{|c|}{ Translation (mm) } & \multicolumn{3}{c|}{ Rotation (arcmin) } \\
\cline { 2 - 7 } & $\Delta \mathrm{V} 1$ & $\Delta \mathrm{V} 2$ & $\Delta \mathrm{V} 3$ & $\Delta \theta 1$ & $\Delta \theta 2$ & $\Delta \theta 3$ \\
\hline Requirement & $+/-1.4$ & $+/-1.0$ & $+/-1.0$ & $+/-0.5$ & $+/-1.1$ & $+/-0.5$ \\
\hline
\end{tabular}

The second requirement for B-to-A, Table 2, limits cooldown motion repeatability for Reference B relative to Reference A.

Table 2. B-to-A Cooldown Motion Repeatability Requirement.

\begin{tabular}{|c|c|c|c|c|c|c|}
\hline BCD Motion Repeatability of & \multicolumn{3}{|c|}{ Translation $(\mathrm{mm})$} & \multicolumn{3}{c|}{ Rotation (arcmin) } \\
\cline { 2 - 7 } BCG wrt ACG & $\Delta \mathrm{V} 1$ & $\Delta \mathrm{V} 2$ & $\Delta \mathrm{V} 3$ & $\Delta \theta 1$ & $\Delta \theta 2$ & $\Delta \theta 3$ \\
\hline Requirement & $+/-0.082$ & $+/-0.056$ & $+/-0.059$ & $+/-0.216$ & $+/-0.215$ & $+/-0.216$ \\
\hline
\end{tabular}

\subsection{SI Pads-to-B Cooldown Performance Requirements}

The thermal distortion limits for science instrument interfaces (SI Pads) relative to Reference B (i.e. internal distortion of the ISIM Structure) is defined in a sub-allocation from the top level SI Pads-to-B alignment budget. This requirement ultimately governs the amount of misalignment that SIs may experience with respect to each other within the Structure upon cooling to the operating temperature and other effects. The overall budget includes terms associated with ambient alignment, cryogenic distortion, gravity release uncertainty, and launch shift. During the Cryoset test, measurements of pads-to-B are compared to the Cryogenic distortion, bare ISIM sub-allocation. This term includes contributions from thermal distortion of the ISIM Structure, desorption, repeatability, and metrology errors. During post-processing, the metrology error due to differences between the optomechanical tooling used to make the SI pads measureable and the actual SI pad interfaces is assessed (Section 4.2). Note that when comparing measured performance against this requirement, consideration of the measurement uncertainty is not required since the measurement uncertainty falls within the metrology sub-allocation from the overall SI Pads-to-B alignment budget. 
Table 3. SI Pads-to-B Cooldown Distortion Requirement.

\begin{tabular}{|c|c|c|c|c|c|c|}
\hline \multirow{2}{*}{ BCD Motion of SI Pads wrt BCG } & \multicolumn{3}{|c|}{ Translation (mm) } & \multicolumn{3}{c|}{ Rotation (arcmin) } \\
\cline { 2 - 7 } & $\Delta \mathrm{V} 1$ & $\Delta \mathrm{V} 2$ & $\Delta \mathrm{V} 3$ & $\Delta \theta 1$ & $\Delta \theta 2$ & $\Delta \theta 3$ \\
\hline Requirement (Cooldown Suballocation) & $+/-0.506$ & $+/-0.506$ & $+/-0.506$ & $+/-5.165$ & $+/-5.165$ & $+/-5.165$ \\
\hline
\end{tabular}

\section{POST-PROCESSING OF TEST RESULTS}

This section describes the post-processing of the metrology data obtained by the photogrammetry system. The metrology dataset consists of spatial coordinates for the metrology targets at ambient and cryogenic temperatures, as well as the change in target coordinates between ambient and cryogenic temperatures.

\subsection{BCG-to-ACG Data Processing}

\section{Approach}

Metrology data characterizing BCG-to-ACG motions must be corrected to remove the effects of ITP cooldown deformations since performance requirements are with respect to an ideal (undeformed) interface. The approach taken to calculate the corrected BCG-to-ACG motion involved three steps. Step 1: Calculate a sensitivity matrix for ISIM BCGto-ACG motion due to ITP-to-KM interface motions. Step 2: Using the sensitivity matrix from Step 1 and the measured ITP-to-KM interface motions calculate the BCG-to-ACG motion associated with ITP deformation. Step 3: Calculate the BCG-to-ACG motion for a perfect ITP/OTE interface by subtracting the ITP deformation contribution from the test measurement.

\section{Kinematic Mount Interface Motion Sensitivity Analysis}

A simple finite element model of the ISIM Structure coupled to the ISIM KMs was used to generate the sensitivity matrix. The model, Figure 4, includes the detailed flight KM model, a simple rigid ISIM including the associated ISIM Reference B targets, a BCG tracking node connected to the eight B targets on the rigid ISIM, and a rigid OTE/ITP interface including the six KM interfaces, Ref A, and ACG tracking nodes. Although the simple model is used for the geometrical alignment change sensitivity study, the detailed flight KM model is the same model used in the integrated flight ISIM thermal distortion analysis. ${ }^{7}$

Using this model, an analysis was performed to calculate BCG-to-ACG motions due to $1 \mathrm{~mm}$ enforced motions of the KM interface points (KMPx, $\mathrm{x}=1$ to 6 ) on a point by point and translational DOF by DOF basis ( 6 interface points $\mathrm{x} 3$ translational DOFs $=18$ cases). Sensitivity studies show that the effect of KM interface point rotations is negligible compared to the effect of interface point translations, so rotation contributions are not considered.

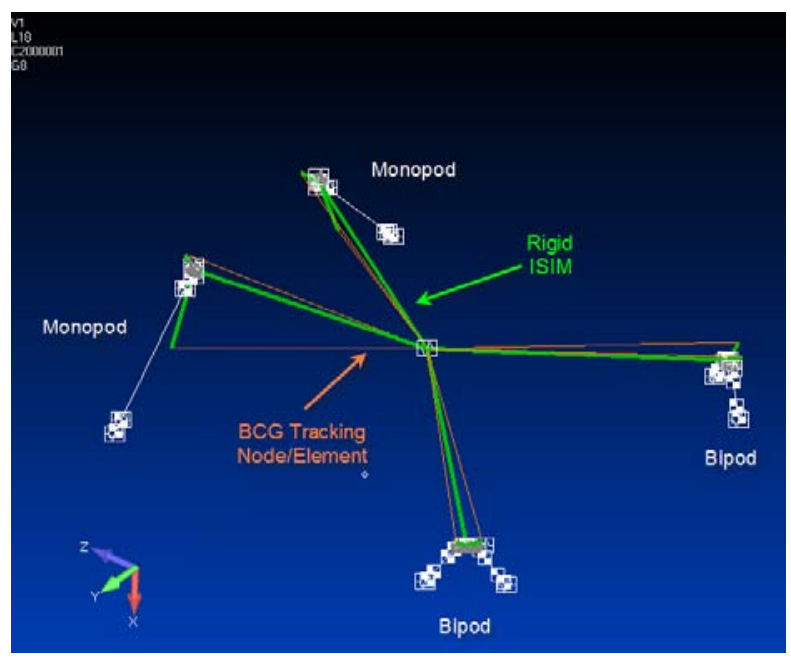

Figure 4. ISIM finite element model used to derive kinematic mount interface motion sensitivity coefficients. 


\section{Interface Deformation Contribution to BCG-to-ACG Motion}

The results of the sensitivity analysis were used to construct an 18x6 sensitivity matrix, $\mathrm{T}$, that provides the BCG-toACG motion of ISIM, $B_{\text {ITP }}$, due to translational perturbations of the KM IPs, $D_{\text {ITP. }}$ The product of this sensitivity matrix and the vector of KM IP translations from the Cryoset test metrology data is used to calculate the ITP deformation contribution to BCG-to-ACG cooldown motions present in the test measurements.

$$
\begin{aligned}
& \left\{B_{\text {ITP }}\right\}=\left\{D_{\text {ITP }}\right\} \cdot\left[T_{\text {ITP }}\right] \\
& \mathrm{B}_{\text {ITP }}=1 \times 6 \text { vector of BCG motions relative to ACG due to ITP deformation } \\
& \mathrm{D}_{\text {ITP }}=1 \times 18 \text { vector of KM IP translations measured during the Cryoset Test } \\
& \mathrm{T}_{\text {ITP }}=18 \times 6 \text { matrix of BCG motion senstivities to KM IP translations }
\end{aligned}
$$

\section{Corrected BCG-to-ACG Motion}

The ITP deformation contribution to BCG-to-ACG motion is subtracted from the BCG-to-ACG measurements from the Cryoset test metrology to obtain the BCG-to-ACG motion associated with the flight ISIM Structure hardware under test.

$$
\begin{aligned}
& \mathrm{B}_{\text {ISIM }}=\mathrm{B}_{\text {Test }}-\mathrm{B}_{\text {ITP }} \\
& \mathrm{B}_{\text {Test }}=\mathrm{BCG}-\text { to }- \text { ACG motions measured during the Cryoset test } \\
& \mathrm{B}_{\text {ITP }}=\mathrm{BCG} \text { - to }- \text { ACG motions due to ITP deformation } \\
& \mathrm{B}_{\text {ISIM }}=\mathrm{BCG} \text { - to }- \text { ACG motions due to flight ISIM Structure hardware deformation }
\end{aligned}
$$

\begin{tabular}{|c|c|c|c|c|c|c|c|}
\hline & & \multicolumn{3}{|c|}{ Translation (mm) } & \multicolumn{3}{|c|}{ Rotation (arcmin) } \\
\hline & PG Target Name & $\Delta \mathrm{V} 1$ & $\Delta \mathrm{V} 2$ & $\Delta \mathrm{V} 3$ & $\Delta \theta 1$ & $\Delta \theta 2$ & $\Delta \theta 3$ \\
\hline \multirow{6}{*}{$\begin{array}{l}\text { PG Measurements for KM IP } \\
(\text { P1-P6) wrt ACG }\end{array}$} & ICDP1 & 0.002 & -0.416 & 0.179 & & & \\
\hline & ICDP2 & 0.002 & -0.314 & 0.321 & & & \\
\hline & ICDP3 & -0.016 & 0.311 & 0.317 & & & \\
\hline & ICDP4 & 0.001 & 0.409 & 0.170 & & & \\
\hline & ICDP5 & -0.002 & 0.426 & -0.493 & & & \\
\hline & ICDP6 & -0.004 & -0.420 & -0.499 & & & \\
\hline \multicolumn{2}{|c|}{ Calculate corrected PG Measurement } & \multicolumn{3}{|c|}{ Translation (mm) } & \multicolumn{3}{|c|}{ Rotation (arcmin) } \\
\hline & Name/Description of Location & $\Delta \mathrm{V} 1$ & $\Delta \mathrm{V} 2$ & $\Delta \mathrm{V} 3$ & $\Delta \theta 1$ & $\Delta \theta 2$ & $\Delta \theta 3$ \\
\hline PG Measurement & BCG wrt ACG & 0.421 & -0.001 & -0.055 & 0.028 & -1.128 & -0.084 \\
\hline PG Measurement Correction & BCG wit ACG & -0.232 & -0.004 & -0.013 & -0.004 & -0.560 & -0.009 \\
\hline $\begin{array}{c}\text { Corrected PG Measurement }= \\
\text { Measurement }- \text { Correction }\end{array}$ & $B C G$ wrt $A C G$ & 0.653 & 0.003 & -0.042 & 0.032 & -0.568 & -0.076 \\
\hline
\end{tabular}

Table 4 presents the measured cooldown deformations of the KM IPs with respect to ACG, the measured total BCG motion with respect to ACG, the calculated BCG-to-ACG correction for measured ITP deformation, and finally the corrected BCG-to-ACG motion representing the BCG-to-ACG motion of the flight hardware under test for the first ambient to cryogenic temperature cycle during the Cryoset test.

Table 4. BCG-to-ACG metrology data post-processing for Cryoset Test Cycle 1, Operational Temperature Cooldown Case.

\subsection{SI Pads-to-BCG Data Processing}

During the Cryoset test, the flight science instrument interface plates that attach to the science instrument kinematic mount feet (SI Pads) are not present and are represented by optomechanical tooling, the science instrument interface plate (SIIP) extender plates (SEPs). Figure 5 depicts the flight SI pad, SIIP, and SEP for the NIRSpec 3 interface. The base of the SEP is identical to the flight SIIP. The SEP includes a post located at the SI interface point coordinates 
(intersection of the KM load line and the interface plane) with the extender plate on top (offset from the interface in the V2 direction). Photogrammetry (PG) targets on the extender plate are used to characterize the translations and rotations of the SI pad interface. The PG target located over the central post is used to characterize SI pad translations. The other PG targets are used to characterize net rotation of the SI pad.

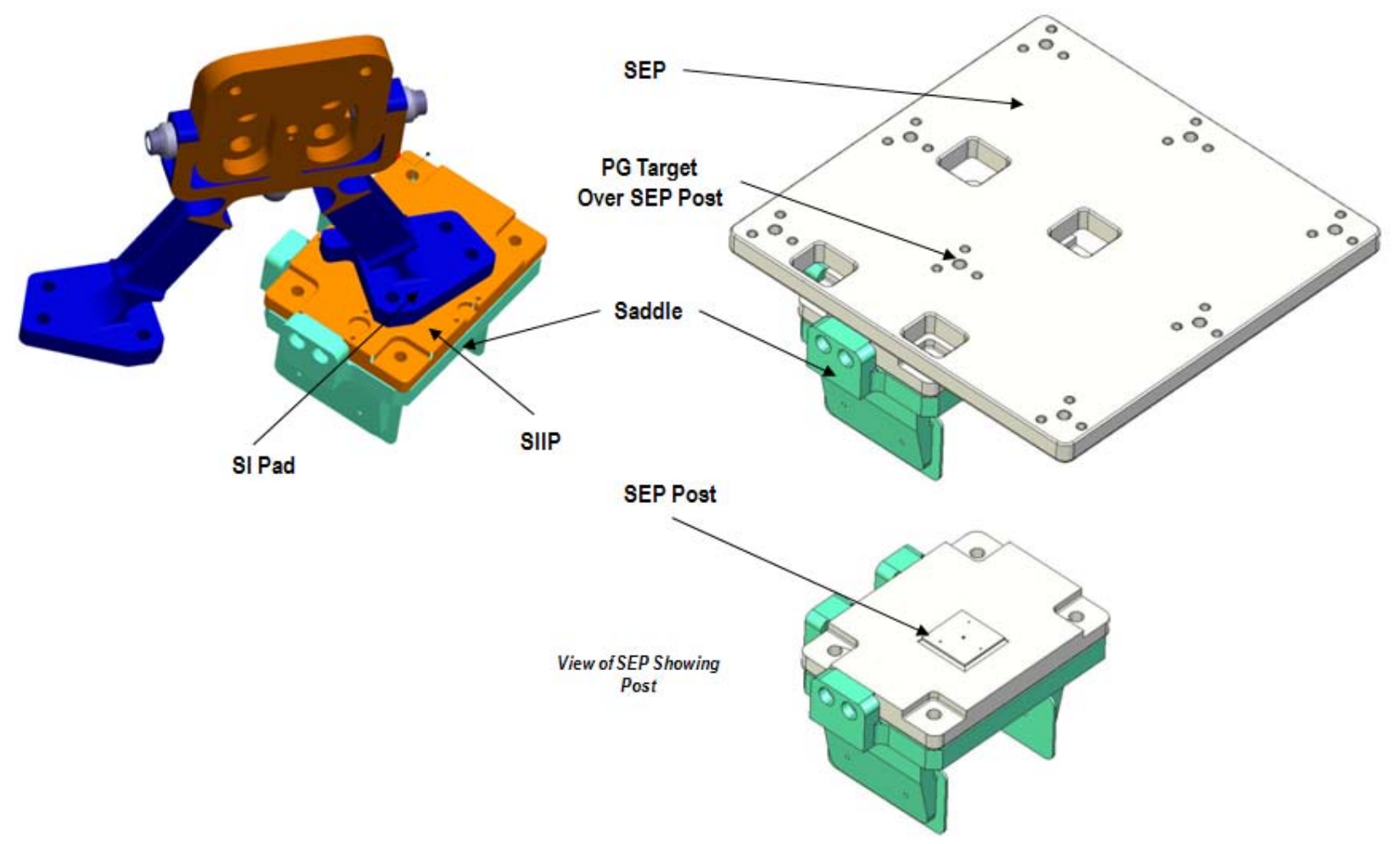

Figure 5. Comparison of Flight SI Pads and SEP tooling used in Cryoset Test.

This optomechanical tooling introduces metrology errors that need to be considered in addition to the photogrammetry measurement uncertainty. The difference between the predicted SEP motions via the PG targets and the predicted SI pad motion at the SI pad to SIIP interface was characterized to assess SEP-SIIP metrology errors. The results of this study indicate that there are two main errors that are introduced by the SEPs. The first error is a simple bias error in V2 associated with the additional pathlength of titanium in the SEPs. The second error is associated with differences in SEP post and SI pad interface footprint geometry coupled with local saddle thermal deformation. The 2-sigma error bars for the PG measurements are $0.03 \mathrm{~mm}$ and 0.5 arcmin due to the PG system alone. Combining the worst SEP/SI pad "tooling errors" on a DOF by DOF basis with the 2-sigma PG error bars in an RSS sense yields a total metrology error that is shown to meet the sub-allocation to metrology errors from the SI Pads-to-B alignment budget. Based on this assessment, no additional post-processing of the SI Pads-to-BCG data from the Cryoset test is necessary for comparison with requirements. However, the V2 bias error was removed from the data since this is a simple, deterministic error to correct. The SEP V2 bias error is equal to the cooldown motion associated with the additional pathlength of titanium. For the nominal $42 \mathrm{~mm}$ pathlength and cooldown from ambient to $39 \mathrm{~K}$, the SEP motion is 68 microns. SI pad motion is obtained by subtracting the V2 bias error equal to the SEP motion from the total measured motion.

\section{COMPARISON OF POST-PROCESSED RESULTS AND REQUIREMENTS}

\subsection{B-to-A Performance}

Post-processed results for BCG-to-ACG motions are compared to the associated optomechanical requirement for Cooldown bulk motion limit in Table 5. Results are presented from the both the ISIM Structure Cryoset and Cryoproof 
Tests. There are a total of four cases: Cryoset Cycle 1 (Ambient to cryo operational temperature, first thermal cycle), Cryoset Cycle 1s (Ambient to cryo cold survival temperature, first thermal cycle), Cryoset Cycle 2 (Ambient to cryo operational temperature, second thermal cycle), and Cryoproof Cycle 1 (ambient to cryo survival temperature, first and only thermal cycle). Note that the results are similar to the order of the measurement uncertainties in all cases. The 2sigma error bars for the photogrammetry measurements for BCG-to-ACG motions are $0.03 \mathrm{~mm}$ and 0.05 arcmin. The measured performance plus associated error bars meets requirements in all cases.

Table 5. Comparison of post-processed BCG-to-ACG measurements with optomechanical Cooldown motion performance requirement.

\begin{tabular}{|c|c|c|c|c|c|c|}
\hline \multirow{2}{*}{ BCD Motion of BCG wrt ACG } & \multicolumn{3}{|c|}{ Translation $(\mathrm{mm})$} & \multicolumn{3}{c|}{ Rotation (arcmin) } \\
\cline { 2 - 7 } & $\Delta \mathrm{V} 1$ & $\Delta \mathrm{V} 2$ & $\Delta \mathrm{V} 3$ & $\Delta \theta 1$ & $\Delta \theta 2$ & $\Delta \theta 3$ \\
\hline Requirement (IST-270) & $+/-1.4$ & $+/-1.0$ & $+/-1.0$ & $+/-0.5$ & $+/-1.1$ & $+/-0.5$ \\
\hline Cryoset Cycle 1 & 0.653 & 0.003 & -0.042 & 0.032 & -0.568 & -0.076 \\
\hline Cryoset Cycle 1s & 0.660 & 0.005 & -0.054 & 0.035 & -0.561 & -0.070 \\
\hline Cryoset Cycle 2 & 0.631 & 0.005 & -0.042 & 0.030 & -0.533 & -0.066 \\
\hline Cryoproof Cycle 1 & 0.667 & -0.005 & -0.045 & 0.038 & -0.515 & -0.038 \\
\hline
\end{tabular}

Post-processed results for BCG-to-ACG motions from the Cryoset Test are compared to the associated optomechanical requirement for bulk Cooldown motion repeatability in Table 6 . Repeatability was calculated be taking the difference between the post-processed BCG-to-ACG motions in Table 5 for the three different cooldown measurement sets (Cycle 1, Cycle 1s, and Cycle 2). The measured performance meets requirements in all cases.

Table 6. Comparison of post-processed BCG-to-ACG measurements from the Cryoset Test with optomechanical cooldown motion repeatability performance requirement.

\begin{tabular}{|c|c|c|c|c|c|c|}
\hline \multirow{2}{*}{$\begin{array}{c}\text { BCD Motion Repeatability of BCG } \\
\text { wrt ACG }\end{array}$} & \multicolumn{3}{|c|}{ Translation (mm) } & \multicolumn{3}{c|}{ Rotation (arcmin) } \\
\cline { 2 - 7 } & $\Delta \mathrm{V} 1$ & $\Delta \mathrm{V} 2$ & $\Delta \mathrm{V} 3$ & $\Delta \theta 1$ & $\Delta \theta 2$ & $\Delta \theta 3$ \\
\hline Requirement (IST-262) & $+/-0.082$ & $+/-0.056$ & $+/-0.059$ & $+/-0.216$ & $+/-0.215$ & $+/-0.216$ \\
\hline Cycle 1 - Cycle 2 & 0.022 & -0.002 & -0.001 & 0.002 & -0.035 & -0.009 \\
\hline Cycle 1 - Cycle 1s & -0.007 & -0.002 & 0.011 & -0.003 & -0.007 & -0.005 \\
\hline Cycle 1s - Cycle 2 & 0.030 & 0.000 & -0.012 & 0.005 & -0.028 & -0.004 \\
\hline
\end{tabular}

\subsection{SI Pads-to-B Cooldown Performance}

Post-processed results for SI pads-to-BCG motions are compared to the associated optomechanical requirement for Cryogenic Bare ISIM distortion in Table 7. In the table, "Max" denotes the numerically largest detected excursion per degree of freedom for all SEPs and similarly, "Min" denotes the numerically lowest excursion per degree of freedom. The requirement was derived such that metrology error is part of the performance requirements, and thus does not need to be added to the measurements for comparison of performance with requirements (i.e., the measurements implicitly include the PG system uncertainty plus the Structure+SEP distortions). The measured performance meets requirements in all cases. 
Table 7. Comparison of SI Pads-to-BCG measurements with optomechanical performance requirement suballocation to Cryogenic Bare ISIM distortion.

\begin{tabular}{|c|c|c|c|c|c|c|c|}
\hline \multirow{2}{*}{\multicolumn{2}{|c|}{ BCD Motion of SI Pads wrt BCG }} & \multicolumn{3}{|c|}{ Translation (mm) } & \multicolumn{3}{|c|}{ Rotation (arcmin) } \\
\hline & & $\Delta \mathrm{V} 1$ & $\Delta \mathrm{V} 2$ & $\Delta \mathrm{V} 3$ & $\Delta \theta 1$ & $\Delta \theta 2$ & $\Delta \theta 3$ \\
\hline \multicolumn{2}{|c|}{$\begin{array}{l}\text { Requirement (IST-263, Table 3-13, } \\
\text { Cryogenic Bare ISIM Suballocation) }\end{array}$} & $+/-0.506$ & $+/-0.506$ & $+/-0.506$ & $+/-5.165$ & $+/-5.165$ & $+/-5.165$ \\
\hline \multirow{2}{*}{$\begin{array}{c}\text { Cycle } 1 \text { (39 K- } \\
\text { Ambient) }\end{array}$} & Max & 0.150 & 0.169 & 0.146 & 1.291 & 0.924 & 0.568 \\
\hline & Min & -0.057 & -0.144 & -0.105 & -1.173 & -0.517 & -1.388 \\
\hline \multirow{2}{*}{$\begin{array}{l}\text { Cycle } 1 \text { ( } 30 \mathrm{~K}- \\
\text { Ambient) }\end{array}$} & Max & 0.160 & 0.169 & 0.149 & 1.396 & 0.921 & 0.405 \\
\hline & Min & -0.059 & -0.150 & -0.111 & -1.209 & -0.511 & -1.394 \\
\hline \multirow{2}{*}{$\begin{array}{c}\text { Cycle } 2 \text { (39 K- } \\
\text { Ambient) }\end{array}$} & Max & 0.134 & 0.163 & 0.124 & 1.402 & 0.899 & 0.561 \\
\hline & Min & -0.043 & -0.153 & -0.083 & -0.707 & -0.402 & -1.131 \\
\hline
\end{tabular}

\section{SUMMARY}

The JWST ISIM Structure successfully completed cryogenic testing in 2010, including the Cryoset Test to characterize and verify cryogenic thermal distortion performance. This paper presented a summary of the post-processing of photogrammetry measurements from the ISIM Structure Cryoset Test for comparison of flight hardware performance with optomechanical requirements for cooldown performance. The post-processed results demonstrate that the ISIM Structure meets all cooldown performance requirements. Future cryogenic thermal vacuum testing of the integrated ISIM Element, consisting of the science instruments and associated subsystems mounted to the ISIM Structure, will build on the success of the ISIM Structure cryogenic testing and verify top-level ISIM thermal and optical performance requirements.

\section{REFERENCES}

[1] Sabelhaus, P.A., Campbell, D., Clampin, M., Decker, J., Greenhouse, M., Johns, A., Menzel, M., Smith, R., Sullivan, P., “An overview of the James Webb Space Telescope (JWST) project,” Proc. SPIE Vol. 5899, p. 241-254, UV/Optical/IR Space Telescopes: Innovative Technologies and Concepts II, (2005).

[2] Greenhouse, M.A., Drury, M.P., Dunn, J.L., Glazer, S.D., Greville, E., Henegar, G., Johnson, E.L., Lundquist, R., McCloskey, J.C., Ohl, R.G., Rashford, R.A., Voyton, M., Status of the James Webb Space Telescope Integrated Science Instrument Module System, Proc. SPIE 7731, 2010.

[3] Kunt, C., Johnston, J.D., Bartoszyk, A., Hendricks, S., "Development and sizing of the JWST Integrated Science Instrument Module (ISIM) metering structure,” Proc. SPIE Vol. 6273, Optomechanical Technologies for Astronomy, (2006).

[4] Bartoszyk, A., Johnston, J.D., Kaprielian, C., Kuhn, J. Kunt, C., Rodini, B., Young, D., "Design/analysis of the JWST ISIM bonded joints for survivability at cryogenic temperatures,” Proc. SPIE Vol. 5868, p. 171-180, Optical Materials and Structures Technologies II, (2005).

[5] Nowak, M.D., Crane, J.A., Davila, P.S., Hylan, J.E., Johnston, J.D., Ohl, R.G., et al., “Cryogenic performance of a high precision photogrammetry system for verification of the James Webb Space Telescope Integrated Science Instrument Module and associated ground support equipment structural alignment requirements,” Proc. SPIE 7793, Optical System Alignment, Tolerancing, and Verification IV, (2010).

[6] Stock, J.M., Connelly, J.A., Nowak, M.D., Wenzel, G.W., Redman, K.W., "Optical metrology of the JWST Integrated Science Instrument Module test platform," Proc. SPIE Vol. 7433: Optical System Alignment, Tolerancing, and Verification III, (2009).

[7] Johnston, J.D., Cofie, E., "An Overview of Thermal Distortion Modeling, Analysis, and Model Validation for the JWST ISIM Structure,” AIAA Structures, Structural Dynamics, and Materials Conference, Denver, CO, AIAA2011-2161, (2011). 